\title{
Cavitation research results of hydroturbine impeller blades and their analysis
}

\author{
Alexey Romanov ${ }^{1}$, Sergey Evdokimov ${ }^{1, *}$, and Vladimir Seliverstov ${ }^{1}$ \\ ${ }^{1}$ Samara State Technical University, Academy of Architecture and Civil Engineering, \\ Molodogvardeyskaya St., 194, Samara, 443001, Russia
}

\begin{abstract}
Cavitation erosion affects the hydropower plants operation mode, destroys the surface of water-conducting way, affects the efficiency coefficient as well as the turbine output. The most common damage is observed on water-conducting ways having poor streamline shape, various ledges, irregularities along the water flow. Disruptive cavitations are formed initially on the rotation axes of vortexes, and the caverns of disruptive cavitation develop with the period subordinating the Strouhal Law. One of the methods of cavitational erosion field studies is the method of obtaining high-speed cavitational erosion of metal plates, which has low resistance to cavitation mechanical effects. It provides an opportunity to determine erosion degree and intensity at different modes of hydropower plants operation. The paper demonstrates the results of studying the cavitational erosion process of hydroturbine impeller blades of Zhiguli Hydroelectric Station (HS). Cavitation tests are conducted for three operating modes. Technological peculiarities are also described. The paper presents results of turbine blades of Unit 5 for three operation modes. The destruction areas of impeller blades peripheral edges are visualized. The study provides the results of field and laboratory cavitation tests of hydroturbine elements that serve as recommendations for repairing and restoration of damaged hydroelectric units of Zhiguli HS.
\end{abstract}

\section{Introduction}

The operation of a hydropower plant is closely connected to the problem of cavitation erosion in water conveyance systems of hydropower plants that arises during operation of hydroelectric units. Questions studying combating cavitation destruction methods have been studied since 1920s [1-4].

There are many causes of cavitation erosion, one of them is the mechanical effect of the water flow in water conveyance systems of hydropower plants [5-8].

Cavitation erosion affects the hydropower plants operation mode, destroys the surface of water-conducting way, affects the efficiency coefficient as well as the turbine output.

\footnotetext{
* Corresponding author: sali5@mail.ru
} 


\section{Materials and methods}

The destruction of the elements surfaces of water-conducting way and the impeller by cavitation erosion influences the operation mode of hydropower plants, the efficiency coefficient as well as the turbine output.

The most common damage is observed on water-conducting ways having poor streamline shape, various ledges, irregularities along the water flow. Disruptive cavitations are formed initially on the rotation axes of vortexes, and the caverns of disruptive cavitation develop with the period subordinating the Strouhal Law.

One of the methods of cavitational erosion field studies is the method of obtaining highspeed cavitational erosion of metal plates, which has low resistance to cavitation mechanical effects. It provides an opportunity to determine erosion degree and intensity at different modes of hydropower plants operation.

\section{Results}

Unit No. 5 was singled out for cavitation tests by high-speed erosion method. The turbine of the unit has six blades made of 20X13 low-alloy stainless steel, being the most resistant to cavitation in any operating conditions of the HS.

The method of performing field cavitation tests by high-speed erosion method is the same as for laboratory studies. At the stopped unit with the drained flowing part of the turbine, an aluminum plate sticking is made. After air drying for two days, the plates are released from the clamps, the unit is started and the corresponding power is selected for the first test mode. Between the start-up of the unit and the selection of the required power, there should be a minimum time for the transient modes not to leave cavitation marks on the plates. The same requirement is also valid for stopping the unit after the tests. After the mode end, the unit must be stopped immediately.

Cavitation tests were carried out for the following cases and modes:

I mode - unit power is $N_{a}=100 \mathrm{MW}$ with a pressure of $H=24,47 \mathrm{~m}$ and tail race level of about $26.70 \mathrm{~m}$.

At this hydro turbines operation mode, according to the head and tail race level, power capability of the unit by cavitation (in concordance with producer factory characteristics) is $109 \mathrm{MW}$. The reduced power of the unit $\mathrm{Na}=100 \mathrm{MW}$ was selected from the condition of a possible additional flooding of the impeller by $1.5 \mathrm{~m}$. As a result of the first mode, the cavitation erosion character in the cavitation free operating mode of the turbine should have been found.

II mode - unit power is $N_{a}=118$ with the head of $H=25,18 \mathrm{~m}$ and tail race level of about $26.20 \mathrm{~m}$.

The power capability is $109 \mathrm{MW}$ by cavitation according to the producer factory characteristics. The unit power is $N_{a}=118$ is selected from the change levels conditions of the tail race at the operational behavior by $1.5 \mathrm{~m}$.

III mode - unit power is $N_{a}=125 \mathrm{MW}$ with a head of $24 \mathrm{~m}$ and tail race level of 27.00 m. As a result of the III mode, cavitation erosion with increased unit power should be detected.

The duration of each mode was 2 hours; the turbine performance was recorded every 0.5 hours. 


\section{Discussions}

Field and laboratory tests were carried out at fixed heads and under the same natural conditions [9].

Analysis of laboratory and field cavitation studies using Zhiguli hydropower plant as an example showed that the intensity of cavitation erosion is the same for both field and laboratory studies. In the boundary layer, there is a movement associated with the motion of the main flow. Water movements affect water bubbles, and they move along the boundary layer.

The ultrasonic noise measured during the cavitation tests indicates the increased sizes of cavitation bubbles of the field turbine. Noise frequency on the field turbine is lower than on the model.

Special attention should be paid to the change regularity of integrated intensity of cavitation radiation, revealed during the tests, at equal heads, but different tail race levels. With a suction head decrease (at higher tail race levels), the intensity of cavitation radiation in the active cavitation zone does not decrease, but increases at equal heads. This is due to the fact that with the rise of tail race mark, the cavitation develops later, the intensity of cavitation continues to grow, and with the rise of tail race level, the pressure in the cavitation caverns closing zone increases.

When choosing a turbine and impeller type, as well as suction head, the need to ensure cavitation free operating conditions at all the modes was taken into account. This requires a significant decrease in the turbine location marking in relation to the tail race.

As a result of testing under different modes, erosion was showed to be in the outlet edge area (near the impeller blades peripheral edge) (Figure 1).

The total erosion zone reached $0.071 \mathrm{~m}^{2}$. Thus, even at cavitation free operation mode of turbine the effects of cavitation erosion appeared on the blades.

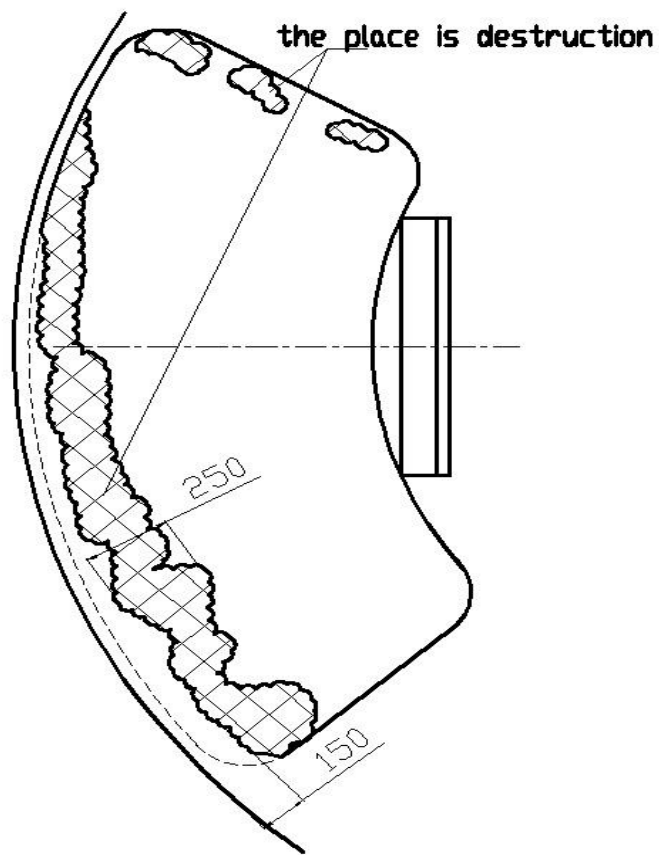

Fig. 1. The destruction areas of impeller blades peripheral edges. 
Studies of other modes showed an increase in cavitation erosion, and the intensity of cavitation erosion remained the same, but its area increased to $0.225 \mathrm{~m}^{2}$, and additional erosion areas were added.

Under proper operation activity and other conditions being equal the appearance of cavitation destructions of the turbine parts will depend on the cavitation resistance of the materials that the hydro turbine is made from. The use of cavitation-resistant materials for any hydro turbine contributes to an increase in its service life and the elongation of an overhaul period. Although timely maintenance work can prevent the occurrence of significant destructions, reduces the time and amount of repair.

The obtained results of field and laboratory cavitation tests of hydro turbine elements serve as recommendations basis for repairing and restoration of damaged hydroelectric units of Zhiguli HS.

Under proper operational conditions of the hydroturbine water conveyance system, excessive destruction as a result of cavitation, which may require the replacement of parts, is not allowed. In most cases, the repair of the water flowing parts of the turbines is carried out on site without assembling. To repair is to prepare, weld and scrape bright all the parts destroyed by cavitation. The surface is prepared by cutting the destructed metal layer to a clean one. Surface welding of impellers blades and chambers made of stainless steel at a depth of no more than $2-3 \mathrm{~mm}$ can be performed without preheating. The larger layer should be repaired with preheating to the temperature of at least $300^{\circ} \mathrm{C}$.

In practice, the cavitation destruction elimination is carried out during major repairs, with an overhaul period of 5 or more years.

At Zhiguli HS 120 impellers blades are installed on 20 turbines, they are made of different steel grades. During operation, stainless steel blades showed slight cavitation destruction. On the blades lined with stainless steel separation and blown facing are observed. Blades made from copper steel were even more unstable to cavitation erosion.

Impellers completing system of one and the same turbine with blades from various steel grades should be recognized as unsuccessful, complicating the production of restoration works and requires more frequent taking the hydraulic unit out of service for repair.

The operational experience of turbines with the details of water conveyance system, made of carbonaceous and low-alloyed steel and reconstructed by means of surfacing of the protective layer by austenitic electrodes, confirmed that this recovery method makes the parts more resistant to cavitation destructions.

A simpler, but less durable way of the impeller chamber surface protecting is its facing with stainless steel strips. The surface is lined with thin strips. The facing process consists of tight pressing and stitching of strips, followed by their welding along the length.

When facing the surface of the impeller, special attention should be given to maintaining the design clearance between the impeller chamber surface and the impeller blades. This can be the reason for the additional cutting down depth of the destructed areas. Surface scraping of the welded metal layer is performed by a pneumatic hammer and a grinding machine.

To preserve the original shape of the parts, surfacing scraping is carried out according to patterns previously removed from a model or made according to the drawings.

The impeller chambers manufacturing from carbon steel with their subsequent facing at the factory with thin stainless sheets leads to significant deformations that arise during the welding process. In this regard, this method was abandoned. It was decided to completely replace the impeller chambers when replacing the latter.

The cavitation study results of hydro turbine impellers have shown that blades made of 20X13low-alloy stainless steel, which can work for the entire period between cavitation repairs of the hydraulic unit, are the most resistant to erosion. The areas of destruction of impeller blades peripheral and trailing edges up to $150 \mathrm{~mm}$ deep may occur during this 
period. These destructions are repaired in the impeller chamber when the blades are deployed. If the gaps between the blades and the impeller acquire a saw tooth shape, and repair by surfacing is impossible, then a special insert can be used to restore them. The section of the blade is cut off and an insert is welded to the blade at this point.

If impeller chamber materials that do not resist to cavitation are used, labor-intensive repairs are required during operation, involving the use of a large number of high-alloy steels and electrodes, and also have additional spares for the repair. Impeller chamber repair is similar to that of blades and shell, which includes the following works: preparatory work, destroyed metal removal, restoration of the original surface shape, surfacing of the protective layer.

\section{Conclusions}

The main conclusions of the paper can be formulated as follows:

1. Field studies of the cavitation process on the impeller blades showed that the maximum pits size in the natural object is more than that on the model. It is explained by the existence of a boundary layer on the blade, where there is a movement connected with water flow movement.

2. Cavitation taking place in turbines, the efficiency and discharge capacity of the impeller are reduced, and separate elements of water conveyance system are destroyed. Cavitation can be detected by cod, noise and shock.

3. The most vulnerable from cavitation point of view are high-speed turbines due to high velocities in the impeller zone and significant decrease in pressure in the suction zone.

4. Manufacture of impellers chambers made of carbon steel and their subsequent facing with thin stainless sheets at the factory leads to significant surface deformations.

5. Reliability of turbines in operation is a decisive quality indicator determined by the average interrepair period. This largely depends on the turbines cavitation properties (cavitation coefficient), as well as cavitation resistance of materials.

6. Turbines operation should be allowed only in modes with low cavitation. The turbine power increase during operation over the design parameter without suction parameter reducing, leads to the cavitation properties reduction, and it is not recommended.

7. Impellers chambers of hydro turbines should be made of stainless steel or bimetallic sheet.

\section{References}

1. V.L. Okulov, I.M. Pylev, Reports of Academ, Power Engineering, 4, 341 (2008)

2. M.I. Balzannikov, M.V. Rodionov, Vestnik MSAU, 2, 70 (2012)

3. M.I. Balzannikov, V.A. Seliverstov, Hydrotechnical Construction, 4, 21 (2012)

4. M.I. Balzannikov, Bulletin of the Department of Building Sciences of RAAES. 2, 209 (2012)

5. M.I. Balzannikov, V.A. Zubkov, N.V. Kondratieva, V.A. Khurtin, Hydraulic engineering construction, 6, 21 (2013)

6. M.I. Balzannikov, M.V. Ivanov, Vestnik MSAU, 12, 139 (2013)

7. M. Balzannikov, M. Rodionov, Proceedings of the Second International Conference GEOTEC. Hanoi, 421 (2013)

8. A.A. Romanov, S.V. Evdokimov, Scientific Review, 7, 188 (2015)

9. A.A. Romanov, S.V. Evdokimov, V.A. Seliverstov, Industrial and civil construction, 6, 44 (2015) 